\title{
THE SYMBIOTIC-BARIUM STARS LINK
}

\author{
V.V. SMITH \\ McDonald Observatory and Dept. of Physics, Univ. of Texas \\ K. CUNHA \\ Observatorio Nacional, Rio de janeiro, Brasil

\begin{abstract}
A. JORISSEN
Institut d'Astronomie et d'Astrophysique, ULB, Belgium

AND

H.M.J. BOFFIN

Dept. of Physics and Astronomy, Univ. of Wales, U.K.
\end{abstract}

An abundance analysis of the yellow symbiotic systems AG Dra and $\mathrm{BD}-21^{\circ} 3873$ reveal them to be metal-poor giants $([\mathrm{Fe} / \mathrm{H}]=-1.3)$ enriched in the heavy s-process elements. The heavy-element abundance distributions of those two stars are almost identical and are best reproduced by a sprocess with a neutron exposure parameter of $1.2-1.3 \mathrm{mb}^{-1}$ and a neutron density $\log N_{\mathrm{n}}=8.3$. These two systems thus link the symbiotic stars to the binary barium and $\mathrm{CH}$ stars which are also s-process enriched. These binary systems, owe their abundance peculiarities to mass transfer from thermally-pulsing asymptotic giant branch stars, which have since evolved to become white-dwarf companions of the cool stars we now view as the chemically-peculiar primaries. We also derive the spectroscopic orbits of those two symbiotic systems and find them similar to those of barium and $\mathrm{CH}$ stars. Finally, because the luminosity function of low-metallicity $\mathrm{K}$ giants is skewed toward higher luminosities by about 2 magnitudes relative to solar-metallicity giants, we argue that lower metallicity $\mathrm{K}$ giants have larger mass-loss rates. It is this larger mass-loss rate that drives the symbiotic phenomena in AG Dra and BD-21 ${ }^{\circ} 3873$. A complete description of this work can be found in Smith et al. (1996, A\&A 315, 179 and 1997, A\&A $324,97)$. 